\title{
Performance Evaluation of Node Failure Prediction QoS Routing Protocol (NFPQR) in Ad Hoc Networks
}

\author{
Dr D Srinivas Rao \\ Professor, Dept of ECE, CE \\ Jawaharlal Nehru Technological University \\ HYDERABAD - 500 085, INDIA \\ dsraoece@gmail.com
}

\author{
Sake Pothalaiah \\ Project Associate, Dept of ECE, CE \\ Jawaharlal Nehru Technological University \\ HYDERABAD - 500 085, INDIA \\ pothalaiahs@gmail.com
}

\begin{abstract}
The characteristics of ad hoc networks make the QoS support a very complex process unlike traditional networks. The nodes in ad hoc wireless networks have limited power capabilities. The node failure in the network leads to different problems such as network topology changes, network partitions, packet losses and low signal quality. Many QoS routing protocols like Predictive location based QoS routing protocol (PLBQR), Ticket based QoS routing, Trigger based distributed QoS routing (TDR) protocol ,Bandwidth routing(BR) protocol, Core extracted distributed routing (CEDAR) protocol have been proposed. However these algorithms do not consider the node failures and their consequences in the routing. Thus most of the routing protocols do not perform well in frequent or unpredictable node failure conditions. Node Failure Predication QoS Routing" (NFPQR) scheme provides an optimal route selection by predicting the possibility of failure of a node through its power level. The NFPQR protocol has been modified as CNFPQR (Clustered NFPQR) in order to provide power optimization using clustered based approach. The performance of the NFPQR and C-NFPQR is evaluated through the same QoS parameters.
\end{abstract}

Keywords- NFPQ; C-NFPQR; CEDAR; PLBQR; TDR; QoS; Routing Protocols;

\section{INTRODUCTION}

Ad hoc Network is a wireless network consisting of a collection of mobile nodes with no fixed infrastructure. Networking infrastructure refers to the facility of which sole purpose is network management and routing. The network nodes communicate with one another over scarce wireless channel in multi hop fashion. Each node behaves as a router and it takes part in discovery and maintenance of routes to other nodes in the network. The main characteristics of this network are dynamic topology, bandwidth constraint, variable capacity links, power constrained operation, limited physical security and quickly deployable. One of the major research issues is related to these networks is quality of service (QoS) routing.

\section{RELATED PROTOCOLS}

Quality of service is set of service requirements provided to certain traffic by the network to meet the satisfaction of the user of that traffic. It has been investigated by different researchers and several proposals have been published to address how the QoS can be supported in MANETs [2]. QoS routing support in MANET still remains as an open problem. In this paper we discuss the related protocols and compare them with our work. The predictive location-based QoS routing protocol (PLBQR) is based on the prediction of the location of nodes in Ad hoc networks. PLBQR protocol uses location and delay prediction schemes which reduce to some extent the problem arising due to the presence of stale routing information. PLBQR has no resources are reserved along the route from the source to the destination, it is not possible to provide hard QoS guarantees using this protocol. Even soft QoS guarantees may be broken in cases when the network load is high[2].

In the trigger-based (on-demand) distributed QoS routing (TDR) protocol [2] due to small-scale fading, the received power level may vary rapidly over short periods of time or distance traveled. Some of the factors that influence fading are multi-path propagation, velocity of the nodes, and bandwidth of the channel.

The bandwidth routing (BR) protocol [2] consists of an end-to-end path bandwidth calculation algorithm to inform the source node of the available bandwidth to any destination in the ad hoc network, a bandwidth reservation algorithm to reserve sufficient number of free slots for the QoS flow, and a standby routing algorithm to reestablish the QoS flow in case of path breaks. The CDMA-over-TDMA channel model that is used in this protocol requires assigning a unique control slot in the control phase of super-frame for each node present in the network. This assignment has to be done statically before commissioning the network. Due to this, it is not possible for a new node to enter into the network at a later point of time. If a particular node leaves the network, the corresponding control slot remains unused and there is no way to reuse such slot(s). and also network needs to be fully synchronized.

CEDAR: the core-extracted distributed routing (CEDAR) protocol has been proposed as a QOS routing protocol. CEADR dynamically establishes a core of the network, and then incrementally propagates link state of stable high bandwidth to the nodes of the core to identify and avoid using congested parts of the network. The core nodes are elected by approximating a minimum dominating set of the ad hoc network. However, it is overhead for CEDAR 


\section{NODE FAILURE PREDICTION QOS ROUTING PROTOCOL (NFPQR)}

Most of the QoS routing protocols proposed previously do not perform well in frequent and /or unpredictable node failure conditions. So QoS routing need a relatively accurate prediction of network's future conditions that is not included in the previous works. This work addresses a new routing algorithm NFPQR NFPQR decreases end to end packet delay and some extent of packet loss by predicting the future power level of a node. It calculates the future condition of a node to make it as next relay node in the path discovery. The estimation of future condition of a node depends on the power level of the node at a particular time [2].

The devices generally are dependent on finite battery sources. Once the battery power is completely consumed then the device will go down i.e., the device is considered as under failure .If the radio interface of the mobile device is not functioning, then all the communications from this device will be stopped .A prediction on node failure helps us in providing better QoS routing for ad hoc networks. Suppose a node, having high probability of failure in the near future due to the lack of sufficient power in the battery and the node is selected as a router to forward the packets, after a few seconds the node will be failed and its communication links with its neighbor will be broken. So it cannot forward the packets and many packets will be lost and these packets have to be regenerated and retransmitted then. Another penalty due to the node failure is that the route discovery process should be performed once again to establish new path that may take few more seconds .During this entire process, the packets will be queued up at the downstream node until a new path is set up.

During certain lower power levels, the signal strength is reduced and delay is increased at the MAC level. Sometimes the network partitioning also occurs due the node failures, where the packet may never reach the destination, if the source and the destination nodes are not in the same partition leads to node failure in the network and causes QoS violation which increases end to end delay, packet loss and network throughput.

In order to solve the problems due to node failure and to support QoS , a new approach is proposed which predicts whether a node will be failed in the near future or not .Before the upstream node is selected as a router to forward the packets ,the downstream nodes predicts whether the upstream node will be failed in the near future or not. The heuristic which is used here is based on the power levels in the battery. Power is consumed during communication and processing or computing. Communication power is much higher than the computing power. In communication power, the transmission power, the power needed to transmit a packet, is much higher than others like receiving power, idle power etc.

If the transmission power is $C_{t}$ and overhead energy is $C_{o}$ then the total power needed to transmit the entire buffer is:

$$
\left(\left(B_{\mathrm{f}} \cdot \mathrm{C}_{\mathrm{t}}\right) / \mathrm{Ps}\right)+\mathrm{C}_{\mathrm{o}}
$$

Here $B_{f}$. is buffer capacity and Ps is packet size.
The threshold power level is based on the packet size, buffer capacity and the packet transfer rate of the node. If $\mathrm{t} 1$ is the present time, then the maximum power consumption at a particular node after time $\mathrm{t} 2$ is given by:

$$
\mathrm{P}_{12}=(\mathrm{t} 2-\mathrm{t} 1) \cdot\left(\operatorname{tr} \cdot \mathrm{C}_{\mathrm{t}}+\mathrm{C}_{\mathrm{o}}\right)
$$

\section{NFPQR ALGORITHM}

When a node $\mathrm{j}$ receives a route request message from a node $\mathrm{i}$, then the node $\mathrm{j}$ predicts its future condition by considering power level of node $j$.

If the power level is above the threshold which is $80 \%$ of the power of the maximum power node in the random network then the node $\mathrm{j}$ will forward the RREQ to the next hop; otherwise it will drop the route request message. The same procedure is repeated for all the nodes till the destination is reached.

\section{Definition (HEAD):}

HEAD nodes are nodes such that all non-HEAD nodes (nodes within the transmission range of that HEAD node) are connected to any one of the HEAD node and route packets for any other nodes with the help of Mobile Agents. The route consists of Source node, Corresponding HEAD node, Gateway nodes and intermediate HEAD and Gateway nodes and destination node.

In this paper our topology management scheme HEAD nodes are selected such that HEAD nodes have maximum power level among their one hop neighbors and all non- HEAD nodes are within the transmission range of HEAD nodes. These HEAD nodes have the routing intelligence i.e. they make all decisions related to routing. The gateway nodes are selected which are having enough/high power so that they can forward packets between HEAD nodes and they don't have routing intelligence.

\section{A. Head Placement}

HEAD nodes along with gateways confirm a path in the virtual backbone, which is used for routing and there is demands for additional power for transmission, reception and processing of packets. Thus the HEAD nodes should be selected in such a way that they have enough/higher power level.

Undecided nodes periodically checks if it has a maximum POWER level among its one-hop neighbors, which have not, joined to any HEAD node (i.e. undecided neighbors). If a node has maximum POWER level among such one hop neighbors, it becomes a HEAD node and declares itself as a HEAD node in the status field of next LINK message and communicates to all its neighbors.

If undecided node knows that its neighbor node has become HEAD node from received LINK message, it changes status to member. It declares its status as member and it is current HEAD node in next LINK message. If more than one neighbors of an undecided node became HEAD, undecided node select its HEAD node from which it has received the 
LINK packet earlier There may be undecided nodes whose one hop neighbors with power level more than the undecided node chose to join HEAD nodes, as the HEAD nodes have more power level than its one hop neighbors. Such undecided nodes with maximum power level among one hop undecided neighbors declares themselves as HEAD nodes in the next LINK message.

A HEAD node prepares a list of its member nodes, which are joined to the HEAD node, form the broadcast of LINK messages received from one hop neighbors. This information in the table is periodically changes as a new LINK packet is received.

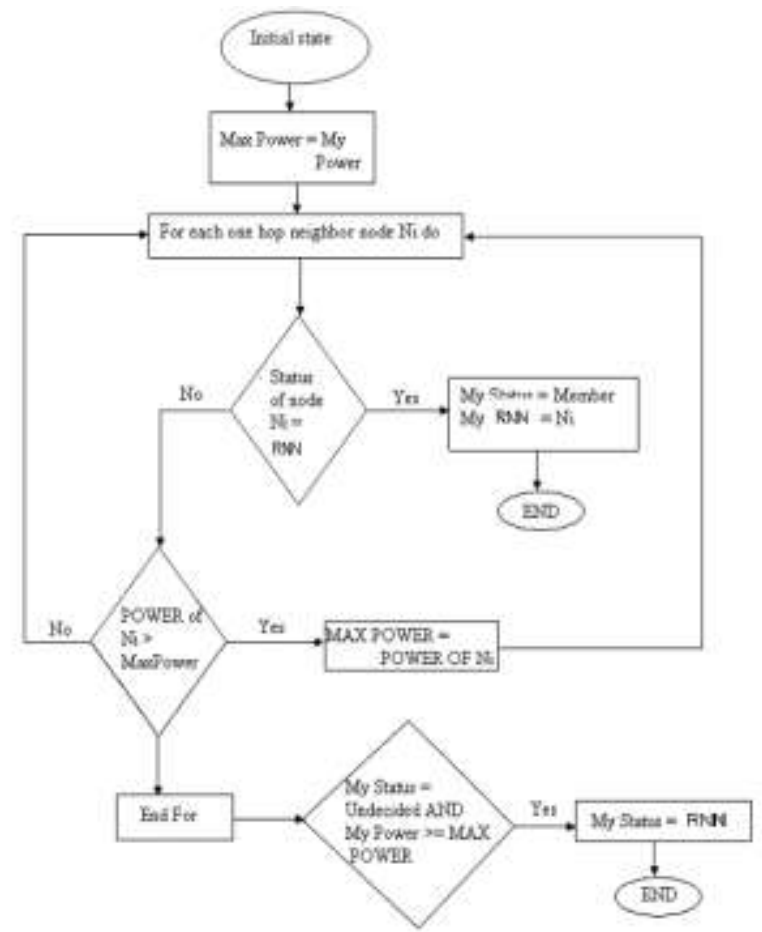

Figure 1. Flowchart for head placement
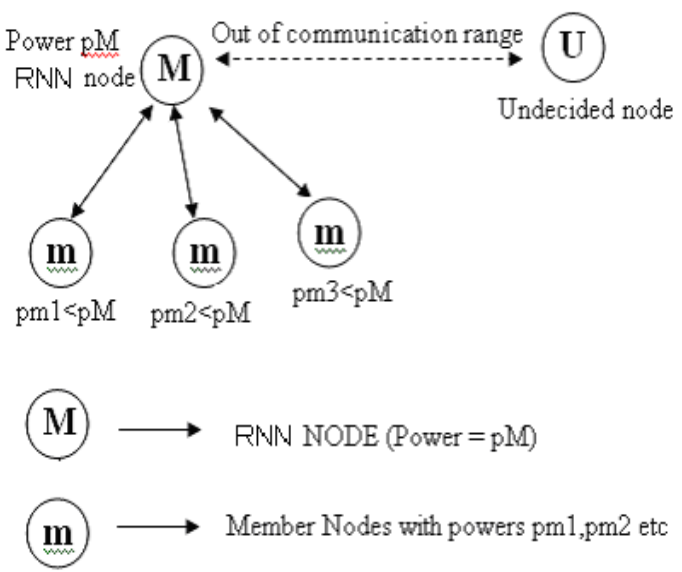

Figure 2. Illustration of HEAD node selection in a Random Ad Hoc Network

\section{B. Head Node Withdrawal}

The HEAD node will drain its energy more rapidly, as compared to member nodes. Before the HEAD node loses its major part of its power, the responsibilities of the HEAD node should be transferred to other node with sufficient power level. Also RIMA nodes should not be changed frequently which will increase the overhead.

When a HEAD node observes that its POWER level is gone below a threshold, it will withdraw its status of HEAD node. The withdrawal of HEAD node is declared to its member nodes in the next WAKEUP message as a undecided node. The threshold can be set to $80 \%$ of HEAD level when the node decided to become a HEAD node.

When a gate way or member node comes to know that it cannot contact its HEAD node, it changes its status to undecided and starts HEAD node placement procedure.

\section{Gateway Selection}

The maximum number of hops between any two close HEAD nodes is two; hence gateways are required and are used to forward the packets between the HEAD nodes. The gateway nodes must have sufficient amount of power, to transmit and receive the packets to and from the HEAD nodes.

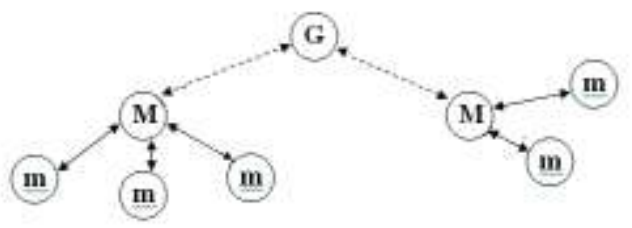

Figure 3. Gateway selection and flow in Ad Hoc Network

$$
\text { M - HEAD Nodes; } m \text { - Member Nodes; G - Gateways }
$$

In NFPQR algorithm, more stable paths are formed during route discovery .Here, the stable path means the packets, which traverses on these paths, will not experience long delays and improves throughput .Also it increases the network life time of the ad hoc networks

\section{Scheduling of Sleep Cycle}

The POWER saving features to 802.11 CSMA/CA to make the MAC layer power efficient by using randomized wake up time for member nodes in ad hoc network. HEAD nodes and Gateways continuously stay awake to forward packets of other nodes

\section{CNFPQR}

Minimizing of power consumption is an important challenge in mobile networking. The requirement of cooperation between power saving and routing protocols is particularly acute in the case of multi-hop Ad hoc wireless networks where nodes must forward packets for each other. Although the quality of routing is improved using NFPQR protocol but it failed to impart power saving mechanism. In 
this paper implementing C-NFPQR (Clustered NFPQR) protocol is implemented over random ad hoc wireless network in which route selection is done using NFPQR protocol. Proposed approach not only optimizes power consumption along with better quality routing leading to better network performances in terms of various QoS parameters

TABLE I. NETWORK SCENARIO

\begin{tabular}{|l|l|}
\hline No .of nodes & 20 \\
\hline Source node id & 14 \\
\hline Destination node id & 8 \\
\hline Network Area & 20X20units \\
\hline Node density & Average \\
\hline Mobility & Random \\
\hline Nodes & Static \\
\hline Power Allocation & Random \\
\hline $\begin{array}{l}\text { Communication } \\
\text { Standard }\end{array}$ & \begin{tabular}{l} 
IEEE \\
\hline
\end{tabular}
\end{tabular}

V. RESUlTS AND DISCUSSION

The mentioned QoS routing approach has been implemented using MATLAB 7.2. The following simulation results obtained during implementation.

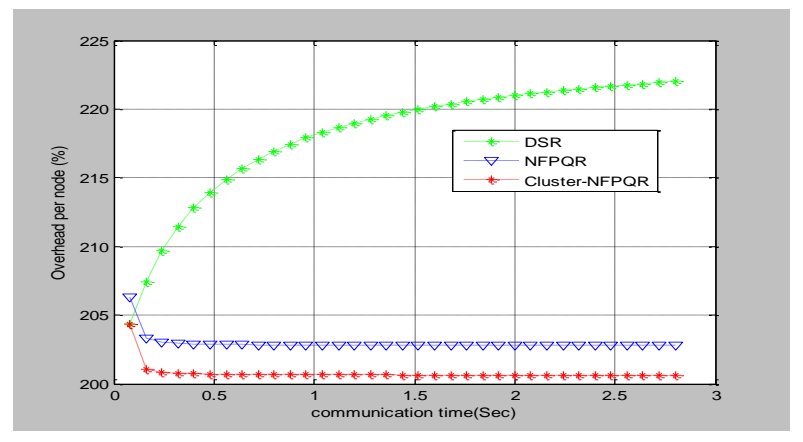

Figure 4. Overhead for DSR, NFPQR and C-NFPQR

The Fig 4 the overhead per node increases with the passage of time for DSR as the nodes drain out of power and the packet needs to be retransmitted through new route. In case of the NFPQR overhead ha reduced a lot as node failure due on the power level basis has been predicted at time of route discovery. This has been further improved using C-NFPQR.

TABLE II. OVERHEAD VALUES

\begin{tabular}{|c|c|c|}
\hline $\begin{array}{c}\text { Routing } \\
\text { protocol }\end{array}$ & $\begin{array}{c}\text { Minimum } \\
\text { overhead }\end{array}$ & $\begin{array}{c}\text { Maximu } \\
\text { m overhead }\end{array}$ \\
\hline
\end{tabular}

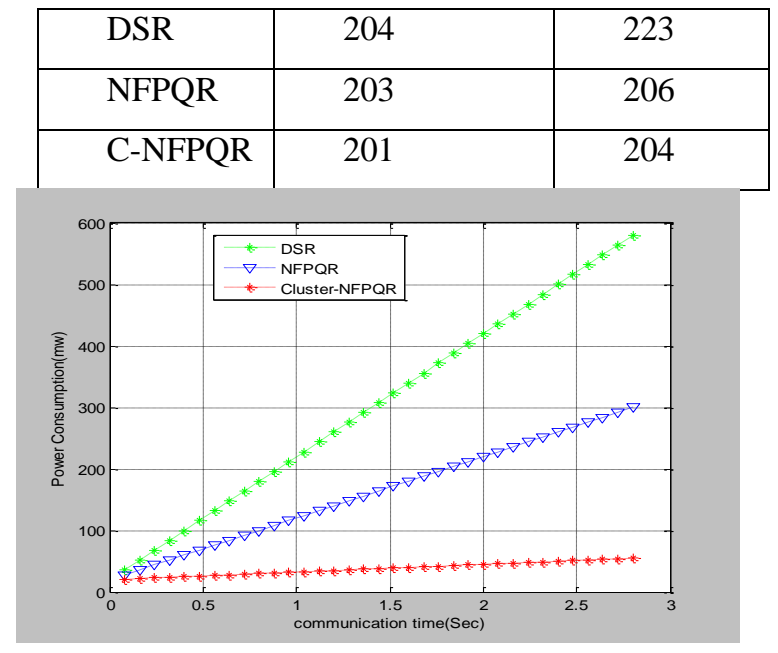

Figure 5. Power consumption for DSR, NFPQR and C-NFPQR

TABLE III. POWER CONSUMPTION RANGE

\begin{tabular}{|l|l|}
\hline $\begin{array}{c}\text { Routing } \\
\text { protocol }\end{array}$ & $\begin{array}{l}\text { Power consumption range } \\
(\mathrm{mW})\end{array}$ \\
\hline DSR & $12-580$ \\
\hline NFPQR & $10-300$ \\
\hline C-NFPQR & $8-60$ \\
\hline
\end{tabular}

As shown in Fig 5 maximum power is consumed using DSR protocol. Power consumption has been reduced further through NFPQR. Though NFPQR does provide power optimization still lesser power consumption accounts to stable routes formed through it. C- NFPQR consumes least power due to power optimization routing

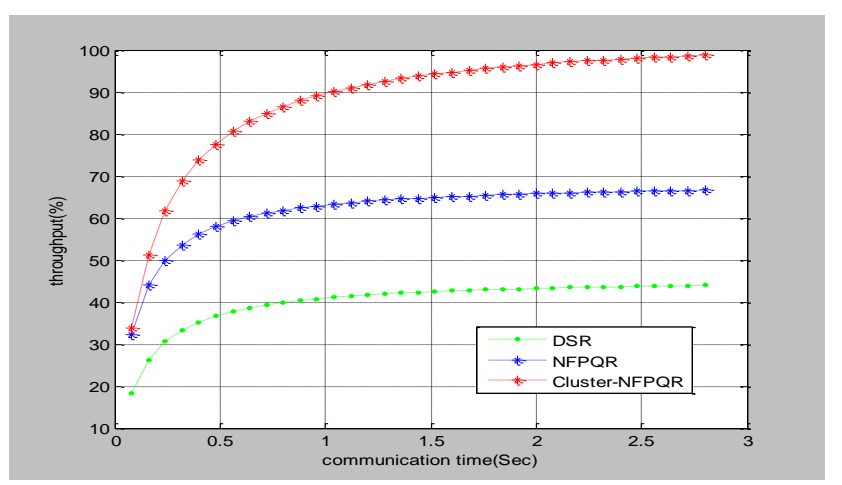

Figure 6. Throughput plot for DSR, NFPQR and C-NFPQR

The above figure Fig 6 shows maximum throughput can be obtained through C-NFPQR protocol. For NFPQR the upper limit for throughput is much less and is least for DSR. Thus in terms of throughput also the performance of C-NFPQR supercedes other two protocols 
TABLE IV. THROUGHPUT RANGE

\begin{tabular}{|c|c|c|}
\hline $\begin{array}{l}\text { Routing } \\
\text { Protocol }\end{array}$ & $\begin{array}{l}\text { Min. } \\
\text { Throughput } \\
\text { T }\end{array}$ & $\begin{array}{c}\text { Max. } \\
\text { Throughput \% }\end{array}$ \\
\hline DSR & 18 & 42 \\
\hline NFPQR & 32 & 68 \\
\hline C-NFPQR & 34 & 98 \\
\hline
\end{tabular}

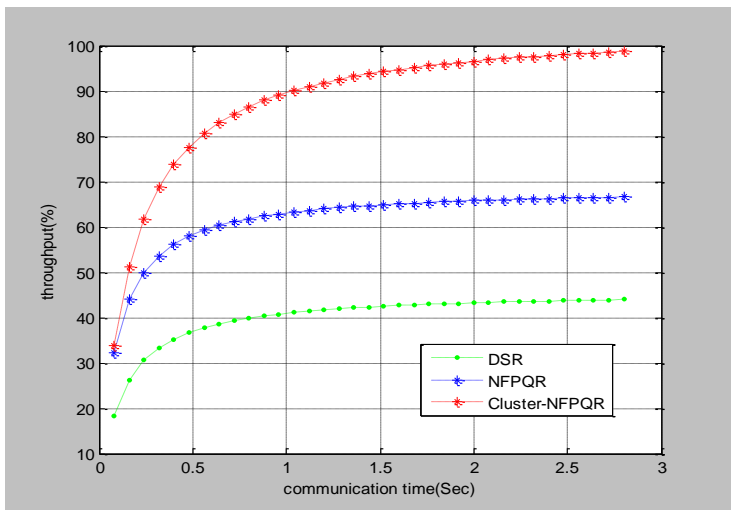

Figure 7. Delay performance plot for DSR, NFPQR and C-NFPQR

From Fig.7 the end to end delay offered to packet communicate from respective source to destination using DSR is almost two times the delay offered using NFPQR, and it comes out to be six times in comparison to C-NFPQR

TABLE V. DELAY RANGE

\begin{tabular}{|c|c|c|}
\hline $\begin{array}{l}\text { Routing } \\
\text { protocol }\end{array}$ & $\begin{array}{l}\text { Minim } \\
\text { um } \\
\text { delay(ms) }\end{array}$ & $\begin{array}{l}\text { Maximum } \\
\text { Delay(ms) }\end{array}$ \\
\hline DSR & 54 & 132 \\
\hline NFPQR & 30 & 70 \\
\hline C-NFPQR & 20 & 24 \\
\hline
\end{tabular}

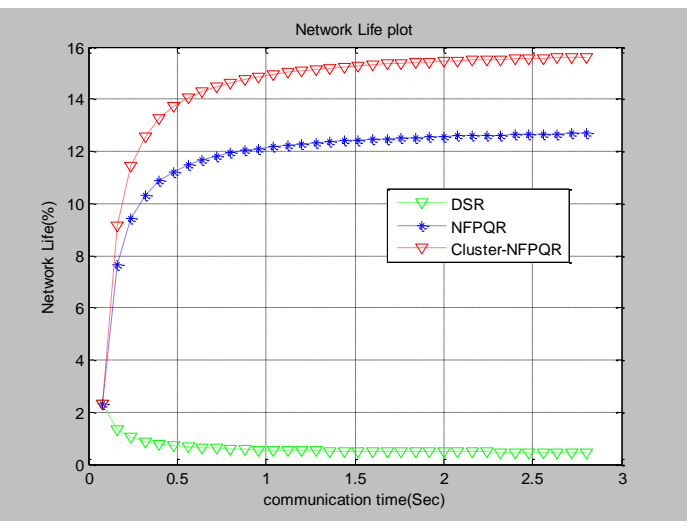

Figure 8. Network plot for DSR, NFPQR and C-NFPQR
From Fig 8 the network life of the ad hoc network has been improved almost ten times through C-NFPQR in comparison to DSR. Though not much significant was improvement reflected in comparison with NFPQR.

TABLE VI. NETWORK LIFE IMPROVEMENT

\begin{tabular}{|c|c|}
\hline $\begin{array}{c}\text { Routing } \\
\text { protocol }\end{array}$ & $\begin{array}{c}\text { Network life } \\
\text { Improvement (\%) }\end{array}$ \\
\hline DSR & 1.6 \\
\hline NFPQR & 10.5 \\
\hline C-NFPQR & 13.8 \\
\hline
\end{tabular}

\section{CONCLUSION}

In this paper performance of C-NFPQR, NFPQR and DSR protocols for random ad hoc network has been evaluated and compared for various QoS parameters. The power level of each node and the respective geographical position is randomly defined in the network. Simulations have been run for 3 seconds considering almost no mobility of nodes during routing and communication. Better QoS routing is provided through NFPQR protocol as it provided stable routes in comparison to DSR due to node failure prediction based on power to some extent improvement in QoS but it fails to impart any power optimization facility. The C-NFPQR overcomes the limitation of both the mentioned approaches by enhancing the performance of the network in terms of evaluated QoS parameters.

\section{REFERENCES}

[1] C.S.R. Murthy and B.S. Manoj, "Ad hoc Wireless Networks: Architectures and Protocols", Prentice Hall, 2004 J. Clerk Maxwell, A Treatise on Electricity and Magnetism, 3rd ed., vol. 2. Oxford: Clarendon, 1892, pp. 68-73.

[2] D.Satynarayana, S.Sathyashree, "Node Failure Predication QoS routing Protocol for ad hoc sensor networks", 2nd International Conference on wireless communication \& sensor networks, December 17-19, 2006.

[3] Tony Larsson and Nicklas Hedman, "Routing Protocols in Wireless Ad hoc Networks -A Simulation Study", Master's thesis in Computer Science and Engineering, Luleå University of Technology Stockholm, 1998.

[4] Mehran Abolhasan,Tadeusz A.Wysocki, and Eryk Dutkiewicz, "A review of routing protocols for mobile ad hoc networks " protocols for mobile ad hoc networks"

[5] David B. Johnson, David A. Maltz, Josh Broch, "DSR: The Dynamic Source Routing Protocol for Multi-Hop Wireless Ad Hoc Networks", Computer Science Department Carnegie Mellon University Pittsburgh.

[6] David B. Johnson, David A. Maltz, "Dynamic Source Routing in Ad Hoc Wireless Networks", Computer Science Department Carnegie Mellon University 5000 Forbes Avenue Pitts burgh.

[7] C.R. Lin and J.S .Liu, "QoS routing in ad hoc wireless networks", IEEE Journal on selected areas in Commuinications", 17: 1426-1438, 1999.

[8] T. Bheemarjuna Reddy, I. Karthigeyan, B.S. Manoj, C. Siva Ram Murthy, "Quality of service provisioning in ad hoc wireless networks: a survey of issues and solutions", Department of Computer Science and Engineering, Indian Institute of Technology, Madras 600036, India , April 14,2004. 
[9] Vikas Kawadia, P. R. Kumar, "Principles and Protocols for Power Control in Wireless Ad Hoc Networks", IEEE journal on selected areas in Communications, vol. 23, no. 1, January 2005.

[10] Shigang Chen and Klara Nahrstedt, "A distributed quality of service routing in ad hoc networks", IEEE Journal on Selected Areas in Commuinications.17 (8), August 1999.

[11] V. Kawadia and P. R. Kumar, "Power control and clustering in ad hoc networks," in Proc. IEEE INFOCOM, 2003, pp. 459-469.

\section{AUTHORS PROFILE}

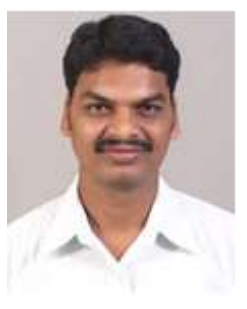

Sake Pothalaiah, graduated from the Department of ECE in National Institute of Technology Waragal in 2006, he obtained his M.E. degree from the department ECE, University College of Engineeering, OU in2008. $\mathrm{He}$ is now working as project Associate, Deprtment of ECE, College of Engineering, JNTU . Kukatpalli

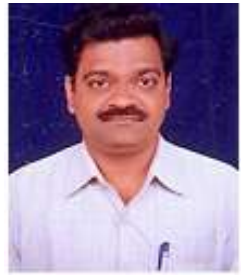

D.Srinivasa Rao, Received the B-Tech degree in Electronics \& Communication Engg from Nagarjuna University, India in 1986 and M.E degree in DigitalSystems, Osmania University, India in 1994, and $\mathrm{PhD}$ degree in Computer Networks from University of Hyderabad, India in 2004. He is now working as Professor, Deprtment of ECE, College of Engineering, JNTU, Kukatpalli, India 